\title{
RadioProphet: Intelligent Radio Resource Deallocation for Cellular Networks
}

\author{
Junxian Huang ${ }^{1}$, Feng Qian ${ }^{2}$, Z. Morley Mao ${ }^{3}$, \\ Subhabrata $\mathrm{Sen}^{2}$, and Oliver Spatscheck ${ }^{2}$ \\ 1 Google Inc. \\ 2 AT\&T Labs - Research \\ ${ }^{3}$ University of Michigan
}

\begin{abstract}
Traditionally, radio resources are released in cellular networks by statically configured inactivity timers, causing substantial resource inefficiencies. We propose a novel system Radioprophet (RP), which dynamically and intelligently determines in real time when to deallocate radio resources by predicting the network idle time based on traffic history. We evaluate RP using 7month-long real-world cellular traces. Properly configured, RP correctly predicts $85.9 \%$ of idle time instances and achieves radio energy savings of $59.1 \%$ at the cost of $91.0 \%$ of signaling overhead, outperforming existing proposals. We also implement and evaluate RP on real Android devices, demonstrating its negligible runtime overhead.
\end{abstract}

\section{Introduction}

Cellular networks employ a specific radio resource management policy distinguishing them from wired and Wi-Fi networks. Previous studies [5][10][8] have shown that in cellular networks, the origin of low resource efficiency comes from the way resources are released. To avoid high signaling load, radio resources are only released after an idle time (also known as the "tail time" or $T_{\text {tail }}$ ) controlled by statically configured inactivity timers. During the tail time, energy is essentially wasted by the radio interface.

Without knowing when network traffic will occur, long tail timer settings (e.g., 11.6 seconds configured by an LTE network [8]) are essentially a conservative way to ensure low signaling overhead, which is known to be a bottleneck for cellular networks. Given that application behaviors are not random, using a statically configured timer is clearly suboptimal. A smaller static timer value helps reduce radio energy, but is not an option due to the risk of overloading cellular networks caused by signaling load increase.

An attractive alternative is to configure the timer dynamically — adaptively performing radio resource release signaled by the handset by monitoring the traffic and accommodating different traffic patterns. But the key challenge is determining when to release resources, which essentially comes down to accurate and efficient prediction of the idle time period. Clearly, the best time to do so is when the handset is about to experience a long idle time period, otherwise the incurred resource allocation overhead (i.e., signaling load) might be unacceptably high. Therefore, accurate and efficient prediction of the idle time period is a critical prerequisite for dynamic timer schemes.

This paper proposes Radioprophet (RP), a practical system running on a handset that makes dynamic decisions to deallocate radio resources based on accurate and efficient prediction of network idle times. It makes the following contributions. 
First, RP utilizes standard online machine learning (ML) algorithms to accurately predict the network idle time, and performs resource deallocation only when the idle time is sufficiently long. We explored various ML algorithms and prediction models with tunable parameters, with the main contribution of using a measurement-driven approach to find robust and easy-to-measure features, whose complex interaction with the network idle time can be automatically discovered by the ML algorithms. The model is validated using seven-month-long traces collected from real users $(\$ 5$ ).

Second, we implement RP on a real Android smartphone to demonstrate its negligible energy and CPU overhead. In contrast, all previous proposals [10][4][7] only perform trace-driven simulation. To reduce the runtime overhead, RP strategically performs binary prediction (i.e., whether the idle time is short or long) at the granularity of a traffic burst consisting of a packet train sent or received in a batch. Compared to fine-grained prediction of the precise value of packet inter-arrival time, our proposed approach is much more efficient while yielding similar optimization results.

Third, we overcome critical limitations of previously proposed approaches, i.e., RadioJockey [4] and MakeIdle / MakeActive [7] are only applicable to background applications without user interaction, with the ideal usage scenario of RadioJockey for a single application only. With multiple concurrent applications, it suffers from low prediction accuracy with increased overhead. In contrast, $\mathbf{R P}$ is specifically designed for both foreground and background traffic. Since its prediction is based on the aggregate traffic of all apps, RP incurs no additional overhead for supporting concurrent apps.

Fourth, we conduct comprehensive measurement of RP using real-world smartphone traces ( 7 months from 20 users). The overall prediction accuracy is $85.9 \%$. RP achieves radio energy saving by $59.1 \%$, at the cost of $91.0 \%$ additional signaling overhead in LTE networks, significantly outperforming previous proposals. To achieve the same energy saving, the additional signaling overheads incurred by MakeIdle [7] and naïve fast dormancy [1] are $305 \%$ and $215 \%$, respectively. The maximal energy saving achieved by RadioJockey [4] is only $27 \%$ since it is only applicable to background traffic.

Paper Organization. We provide sufficient background in $\S 2$ before giving an overview of the Radioprophet (RP) system in $\sqrt{3}$. We detail how we select relevant features for idle time prediction in 4 , and then systematically evaluate RP in $\$ 5$ In $\$ 6$ we describe related work before concluding the paper.

\section{Background}

In cellular networks, there is a radio resource control (RRC) state machine that determines radio resource usage based on application traffic patterns, affecting device energy consumption and user experience. Conceptually similar RRC state machines exist in different types of cellular networks from $2 \mathrm{G}$ to $4 \mathrm{G}$ LTE. In $3 \mathrm{G}$ UMTS networks, there are usually three RRC states [11]: idle, low-power state, and high-power state. In 4G LTE networks, there are only two RRC states: idle and active [8]. Note that RP works for any type of RRC state machine with fast dormancy (described soon) support.

State Transitions. There are two types of state transitions. State promotions switch from a low-power state to a high-power state. They are triggered by user data transmission in either direction. State demotions go in the reverse direction, usually triggered 
by inactivity timers configured by the radio access network (RAN). For example, for a commercial LTE network [8], at the active state, the RAN resets the timer to a constant threshold $T_{\text {tail }}=11.6$ seconds whenever it observes any data frame. If there is no user data transmission for $T_{\text {tail }}$ seconds, the timer expires and the state is demoted to idle. Similar timers exist in $3 \mathrm{G}$ networks (e.g., 12 seconds [11]).

State promotions incur long "ramp-up" delays of up to several seconds during which tens of control messages are exchanged between the handset and the RAN for resource allocation. Excessive state promotions increase the signaling overhead at the RAN and degrade user experience, especially for short data transfers [3][10]. On the other hand, state demotions incur tail times ( $\left.T_{\text {tail }}\right)$ causing waste of radio resources and handset energy [5]. During the tail time, no data is transferred but the handset radio power is much higher than that at the idle state (e.g., $1060 \mathrm{~mW}$ vs $11 \mathrm{~mW}$ for LTE [8]).

Fast Dormancy. Why are tail times necessary? First, the overhead of resource allocation (i.e., state promotions) is high and tail times prevent frequent allocation and deallocation of radio resources. Second, the RAN has no easy way of predicting the network idle time of a handset, so it conservatively appends a tail to every network usage period. This naturally gives rise to the idea of letting the handset actively request for immediate resource release. Based on this intuition, a feature called Fast Dormancy has been included in 3GPP since Release 7 [1][2]. It allows a handset to send a control message to the RAN to immediately demote the RRC state to idle (or a hibernating state) without experiencing the tail time. Fast dormancy is supported by many handsets [2]. It can dramatically reduce the radio resource and the handset energy usage with the potential penalty of increased signaling load when used aggressively [3][10].

\section{The RadioProphet (RP) System}

The static tail times are the root cause of low resource efficiency in cellular networks. RP leverages fast dormancy to dynamically determine when to release radio resources.

Challenge 1: trading off between resource saving and signaling load. The best time to perform resource deallocation is when the handset is about to experience a long idle time period $t$. If $t$ is longer than the tail time, deallocating resources immediately saves resources without any penalty of signaling load (i.e., state promotions). Otherwise, doing so incurs an additional state promotion. Balancing such a critical tradeoff requires predicting the idle time between data transfers so that fast dormancy is only invoked when the idle time is sufficiently long.

Challenge 2: handling both foreground and background traffic. Idle time prediction is particularly difficult for applications involving user interactions. Previous systems, such as RadioJockey [4] and MakeActive [7], simply avoid this by only handling traffic generated by applications running in the background.

Challenge 3: trading off between prediction accuracy and system performance. RP is a service running on a handset with limited computational capabilities and more importantly, limited battery life. So we need to minimize the overhead without sacrificing much of the prediction accuracy. 
To address Challenge 1, we establish a novel machine-learning-based framework for idle time prediction. Besides measuring the effectiveness and efficiency of a widerange of ML algorithms, our key contribution is addressing the hard problem of selecting discriminating features that are relevant to idle time prediction. Based on extensive measurement, we find that strategically using a few simple features (e.g., packet direction and size) leads to high prediction accuracy (4). To address Challenge 2, we designed a general prediction framework that works for the aggregated (possibly concurrent) traffic containing both foreground and background traffic. In contrast, previous systems such as RadioJockey have the ideal usage case for a single app. Further, we leverage the screen status [9], which indicates whether a user is interacting with the device, to customize the prediction for screen-on and off traffic. Such a novel approach can better balance the aforementioned tradeoff between resource saving and signaling load. To address Challenge 3, RP performs binary prediction at the granularity of a traffic burst consisting of a train of packets. In other words, we find that the knowledge of whether the inter-burst time (IBT) is short or long (determined by a threshold) is already accurate enough for guiding the resource deallocation. Such an approach is much more efficient while yielding similar accuracy compared to the expensive approach of predicting the precise value of packet inter-arrival time.

RP consists of three components: a traffic monitor, an IBT prediction module, and a Fast Dormancy (FD) scheduler. The monitor inspects network traffic (only examines packet headers) and extracts lightweight features for each burst in an online manner. The features are then fed into the IBT prediction module, which trains models to predict the IBT for the current burst. Then, the FD scheduler makes decision on whether to invoke fast dormancy based on the IBT prediction result.

For Івт prediction, we formulate the traffic pattern as follows. The traffic is a sequence of packets $\left\{P_{i}\right\}(1 \leq i \leq n)$ in both directions. Let the timestamp of $P_{i}$ be $t_{i}$. Using a burst threshold BT, the packets are grouped into bursts, i.e., $\left\{P_{p}, P_{p+1}, \ldots, P_{q}\right\}$ belongs to a burst $B$ if and only if: (1) $t_{k+1}-t_{k} \leq \mathbf{B T}$ for $\forall k \in\{p, \ldots, q-1\}$, (2) $t_{q+1}-t_{q}>\mathbf{B T}$, and (3) $t_{p}-t_{p-1}>\mathbf{B T}$. We define the inter-burst time IBT of burst $B$ to be the time gap following this burst, i.e., $t_{q+1}-t_{q}$. We use a short IBT threshold called SBT to classify an IBT, i.e., if IBT $\leq \mathbf{S B T}$, the burst is short, otherwise, it is long.

The IBT prediction module trains a model based on historical traffic information, which consists of an array of bursts $\left\{B_{1}, \ldots, B_{m}\right\}$. Each $B_{i}$ is a vector $\left(f_{1}, f_{2}, \ldots, f_{t}\right.$, $\left.i b t_{i}\right)$ where $\left\{f_{1}, \ldots, f_{t}\right\}$ is the list of features of $B_{i}$ and $i b t_{i}$ is the IBT following burst $B_{i}$ observed by the traffic monitor. Whenever there is an idle time of BT, i.e., a new bust appears, the prediction process starts. The feature vector of the current burst $\left\{f_{1}, \ldots, f_{t}\right\}$ is generated and fed to the prediction module, which predicts whether the IBT following the current burst is short or long. If short, no change is made and the handset stays in the tail, since a packet is likely to appear soon. Otherwise, the FD scheduler invokes fast dormancy to save energy. The prediction model is customized for each handset, and is dynamically updated to adapt to the recent traffic pattern.

\section{Feature Selection}

We describe the measurement dataset before studying the feature selection in 4.2 


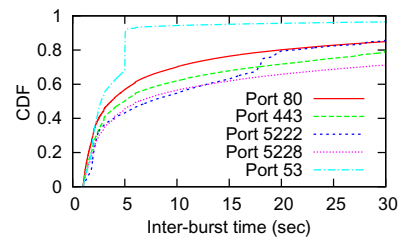

Fig. 1. IBT distributions of bursts whose last packets have specific port numbers

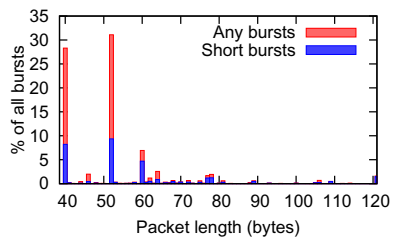

Fig. 2. Distributions of bursts grouped by packet length of the last packet

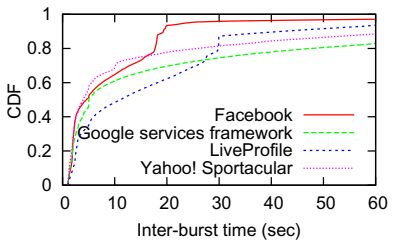

Fig.3. IBT distributions of bursts whose last packets are associated with specific apps

\subsection{The UMICH Dataset}

The measurement data used in this study, which we call the UMICH dataset, is collected from 20 students at University of Michigan for seven months. The students were given Motorola Atrix (11 of them) or Samsung Galaxy S smartphones (9 of them) running Android. Our custom data collection software continuously runs in the background and collects three types of data. (1) Packet traces (only headers are used in this study). (2) The process name responsible for sending or receiving each packet. (3) Other system information such as screen status. Over the seven months (May to Dec 2011) we collected 152 GB data. Although both cellular and Wi-Fi traces were collected, in this study, we only use cellular traces, which contribute to $57.8 \%$ of the total traffic volume.

\subsection{Measurement Driven Feature Selection for Burst Classification}

We use a measurement-driven approach to derive features for the prediction model by analyzing the correlation between various features and the IBT. First, to predict whether an IBT is short or long, we look at the burst right before the IBT, since we observe that the correlations between the IBT and earlier bursts' features are much weaker. Second, the features are extracted from the last three packets of a burst. This is because in most cases, bursts are small (53\% of bursts consist of no more than 3 packets), and even for large bursts, we can usually tell their nature based on the last three packets, e.g., TCP three-way handshake. Third, we only inspect packet headers since examining payload incurs much higher overhead and also because traffic is increasingly being encrypted.

The lightweight features of the last three packet 1 used by RP are listed below: (1) packet direction, (2) server port number, (3) packet length (including header), (4) protocol field in IP header, (5) TCP flags field in TCP header (0 if not TCP), and (6) application name associated with the packet. These features are selected empirically so that they are most relevant to IBT based on our measurement. We show three features below as examples. We start our analysis with $\mathbf{B T}=1 \mathrm{~s}$ and $\mathbf{S B T}=3 \mathrm{~s}$. Later we explore how different BT and SBT settings affect our results in a quantitative manner $(\$ 5.4)$.

Port Number. Figure 1 shows IBT distributions of the top 5 ports ordered from top to bottom in the legend, e.g., 80 is the most popular port, across all users. IBT distributions

\footnotetext{
${ }^{1}$ If a burst contains less than three packets, all features for the missing packet(s) have a value of 0 .
} 
of different ports clearly differ, especially for port 53 , whose sudden jump at IBT $=5$ seconds corresponds to the DNS retransmission timeout on Android. We also observe clusters of Iвт values for many other ports. For example, most bursts over port 5222 have a 20 -second Iвт corresponding to the keep-alive periodicity of Facebook.

Packet Length. Figure 2 plots the distributions of last packet lengths of bursts with short IBT (IBT < SBT) and all bursts. Most bursts end with small packets, i.e., $84.59 \%$ have their last packets $\leq 100$ bytes, as a large packet is typically in the middle of a burst. We observe high correlation for a few packet lengths values. For example, for 121 bytes, $93.04 \%$ bursts have short IBTs. The machine learning algorithms could automatically discover these rules for prediction.

Applications. In Figure 3, the legend shows the sorted list of apps contributing the largest amount of bursts with Facebook ranked at top 1. The differences in IBT values are clear across apps. We also observe that for some apps, their periodic transfer behaviors contribute to clusters of specific Iвт values, e.g., Facebook and LiveProfile. The application information can be very efficiently obtained (e.g., on Android [11]).

\section{Implementation and Evaluation}

\subsection{Implementation}

Trace-Driven Evaluation. We implement simulators of RP, MakeIdle [7], and RadioJockey [4] on a desktop (3.16 GHz Xeon CPU with 16GB memory) using Matlab. They work with an RRC state machine simulator (\$5.2). We use them to evaluate the accuracy and resource savings of RP under various configurations (\$5.3 \$5.4), as well as to compare RP with other optimization techniques $(\$ 5.5)$, using the UMICH trace.

Implementation on Real Android Phone. We also implement the full RP system on a Samsung Galaxy S3 phone running Android 4.0.4 to evaluate its running overhead (\$5.6). A modified TcpDump program is used as the traffic monitor. The IBT prediction module is implemented as a native Android application running in the background.

\subsection{Evaluation Methodology}

We use three metrics to evaluate RP: prediction accuracy, saved radio energy, and increased signaling load. The accuracy is defined as the number of bursts whose immediate IBT (short or long) are correctly predicted divided by the total number of bursts in the input trace. The radio energy, denoted as $E$, is the energy consumed by the handset radio interface. It is one of the most significant components for the overall energy usage of a handset, along with screen and CPU energy [11]. We build an RRC state machine simulator, which takes as input a packet trace and employs the LTE radio energy model derived in our previous work [8] to calculate $E$ (using a UMTS model [11] yields qualitatively similar results). The signaling load, denoted as $S$, is quantified by the number of state promotions, each incurring a fixed number of signaling messages [4]. $S$ is also computed by the RRC state machine simulator.

Assume when a specific user trace is evaluated without any optimization performed (no fast dormancy), $E$ and $S$ are calculated to be $E_{d}$ and $S_{d}$, respectively. When RP 
Table 1. Impact of $\alpha, \beta$ on the prediction accuracy (PerUserDynamic model)

\begin{tabular}{|c|c|c|c|c|c|}
\hline$\alpha=$ & 100 & 500 & 1000 & 2000 & 5000 \\
\hline$\beta=1$ & $81.5 \%$ & $83.7 \%$ & $\mathbf{8 4 . 2 \%}$ & $82.4 \%$ & $80.2 \%$ \\
\hline$\beta=2$ & $80.1 \%$ & $81.4 \%$ & $82.9 \%$ & $82.0 \%$ & $80.0 \%$ \\
\hline$\beta=5$ & $79.8 \%$ & $80.9 \%$ & $81.4 \%$ & $81.0 \%$ & $79.3 \%$ \\
\hline$\beta=10$ & $79.4 \%$ & $80.0 \%$ & $80.9 \%$ & $80.0 \%$ & $79.0 \%$ \\
\hline$\beta=20$ & $78.9 \%$ & $79.6 \%$ & $80.2 \%$ & $79.5 \%$ & $78.7 \%$ \\
\hline
\end{tabular}

Table 2. Summary of prediction models

\begin{tabular}{|c|l|c|}
\hline Name & Description & Accuracy \\
\hline $\begin{array}{l}\text { Peruser } \\
\text { Dynamic }\end{array}$ & $\begin{array}{l}\text { Use most recent } \alpha \text { bursts of a user } \\
\text { to predict next } \beta \text { bursts for that user }\end{array}$ & $84.2 \%$ \\
\hline $\begin{array}{c}\text { Peruser } \\
\text { Static }\end{array}$ & $\begin{array}{l}\text { Use a fixed set of } n \text { bursts of a user } \\
\text { to train a fixed model for that user }\end{array}$ & $80.8 \%$ \\
\hline $\begin{array}{c}\text { Alluser } \\
\text { Static }\end{array}$ & $\begin{array}{l}\text { Use a fixed set of } k \text { bursts of all users } \\
\text { train a fixed model for all users }\end{array}$ & $77.5 \%$ \\
\hline
\end{tabular}

is used, the resulting $E$ and $S$ become $E^{\prime}$ and $S^{\prime}$, respectively. We define $\Delta(E)=$ $\left(E_{d}-E^{\prime}\right) / E_{d}$ and $\Delta(S)=\left(S^{\prime}-S_{d}\right) / S_{d}$ (usually both are positive). They correspond to the reduction of the radio energy and the increase of the signaling load brought by $\mathbf{R P}$, respectively. RP's goal is to maximize $\Delta(E)$ while minimizing $\Delta(S)$.

\subsection{Prediction Model Comparison}

In RP, we use recent traffic information of a user to train a model, denoted as PerUserDynamic. Specifically, for each user, the most recent $\alpha$ bursts are used to predict the next $\beta$ bursts. We study the impact of $\alpha, \beta$ in Table 1 using the Ensemble Bagging [6] learning algorithm as an example (number of trees set to 20). If $\alpha$ is too small, there is not enough training data for learning; if $\alpha$ is too large, the user is more likely to switch to new applications that generate different traffic patterns so previously learned rules may not be useful. Based on Table 1 we choose $\alpha=1000$ and $\beta=1$ that maximize the accuracy. In practice, $\alpha$ and $\beta$ could also be dynamically adjusted.

Table 2 compares the PerUserDynamic model with two other models, PerUserstatic (a fixed model for each user) and Alluserstatic (a fixed model for all 20 users). For fair comparison, we use the same ML algorithm (Ensemble Bagging) as used in Table 1. We set $\alpha=1000$ and $\beta=1$ for the PerUserDynamic model as discussed previously, and use $n=10,000$ for PerUserstatic and $k=10,000$ for Alluserstatic ( $n$ and $k$ defined in Table 2). Similar to Table $1 n$ and $k$ are empirically selected to yield good prediction accuracies. We observe that PerUserDynamic has higher prediction accuracy than the other two models, suggesting that it is necessary to have a dynamic model for each user whose traffic pattern may be different from others.

\subsection{Selecting Burst Thresholds}

We study the impact of $\mathbf{B T}$ and $\mathbf{S B T}$ (previous evaluations use $\mathbf{B T}=1 \mathrm{~s}$ and $\mathbf{S B T}=3 \mathrm{~s}$ ). In Table $3 S_{0}$ to $S_{4}$ correspond to representative (BT, SBT) pairs. We find that aggressively using a short SBT $\left(S_{1}\right)$ can significantly increase $\Delta(S)$. Among all settings, $S_{4}$ yields the highest $\Delta(E) /(1+\Delta(S))$ value (the average radio energy saving per unit of signaling load). It quantifies how well the balance between $\Delta(E)$ and $\Delta(S)$ is handled.

As mentioned in $\$ 3$, configuring screen-on and off settings differently may yield better optimization results, as screen-off traffic is usually generated by background apps without user interaction, leading to statistically longer IBT. Therefore a more aggressive 
Table 3. Impact of BT and IBT (Classification Tree with PerUserDynamic model, $\alpha=1000, \beta=1$ )

\begin{tabular}{|c|c|c|c|c|}
\hline Settings (unit: sec) & Accuracy & $\Delta(E)$ & $\Delta(S)$ & $\frac{\Delta(E)}{(1+\Delta(S))}$ \\
\hline$S_{0}$ BT: 1 SBT: 3 & $82.65 \%$ & $52.10 \%$ & $101.64 \%$ & 0.26 \\
\hline$S_{1}$ BT: 1 SBT: 2 & $84.80 \%$ & $56.69 \%$ & $158.99 \%$ & 0.22 \\
\hline$S_{2}$ BT: 1 SBT: 4 & $81.94 \%$ & $49.07 \%$ & $83.34 \%$ & 0.27 \\
\hline$S_{3}$ BT: 0.5 SBT: 3 & $84.71 \%$ & $53.74 \%$ & $100.36 \%$ & 0.27 \\
\hline$S_{4}$ BT: 1.5 SBT: 3 & $85.39 \%$ & $58.85 \%$ & $93.75 \%$ & 0.30 \\
\hline $\begin{array}{c}S_{5} \text { BT: } 1 / 1.5 \text { off/on } \\
\text { SBT: } 2.5 / 3 \text { off/on }\end{array}$ & $85.88 \%$ & $59.07 \%$ & $91.01 \%$ & 0.31 \\
\hline
\end{tabular}

Table 4. Performance and accuracy of different ML algorithms

\begin{tabular}{|c|c|c|}
\hline $\begin{array}{c}\text { ML } \\
\text { Algorithm }\end{array}$ & $\begin{array}{c}\text { Prediction time } \\
\text { (Training time) }\end{array}$ & Accuracy \\
\hline Naïve Bayes & $\begin{array}{c}2.5 \mathrm{~ms} \\
6.4 \mathrm{~ms}\end{array}$ & $76.1 \%$ \\
& $5.9 \mathrm{~ms}$ & $85.9 \%$ \\
\hline Classification & $136.9 \mathrm{~ms}$ & \\
Tree & $106.6 \mathrm{~ms}$ & $87.4 \%$ \\
\hline Ensemble & $626.1 \mathrm{~ms}$ & \\
Bagging & \multicolumn{2}{|l}{} \\
\hline
\end{tabular}

setting (smaller BT and SBT) can be applied to screen-off traffic without incurring much signaling overhead. In Table 3, $S_{5}$ is such a screen-aware setting. Compared with $S_{4}$, $S_{5}$ saves more energy with less signaling overhead incurred. In fact, $S_{5}$ achieves results comparable to the optimal scenario to be shown in Table 5. This also indicates that dynamically changing BT and SBT can help improve the effectiveness of RP.

\subsection{Comparing Fast Dormancy Based Resource Optimization Approaches}

Table 5 compares various optimization techniques using the UMICH dataset.

Basic fast dormancy. We set $T_{\text {tail }}$ to a fixed value smaller than its original value.

RadioJockey [4] uses system calls to predict the end-of-session (EOS) for background app without user interaction, with the ideal usage scenario for a single app. Given that we do not have system call traces in our dataset, we make two assumptions in our simulation: (1) we use end-of-burst to approximate end-of-session, (2) RadioJockey has high prediction accuracies $(90 \%$ and $100 \%)$ for both single and concurrent apps (although in reality, it performs worse when concurrent apps exist). A key limitation of RadioJockey is it does not handle foreground traffic and only works when the screen is idle (see 96 ), so we only apply RadioJockey to screen-off traffid2.

MakeIdle [7] computes a wait time $T_{\text {wait }}$ that maximizes the energy saving if $T_{\text {tail }}$ is set to $T_{\text {wait }}$ for the previous $M$ packets, it then applies this $T_{\text {wait }}$ for the next $N$ packets. The range we search for the optimal $T_{\text {wait }}$ is $[0.5,11.5]$ seconds, as suggested by the authors. Since no recommendations have been made for the values of $M$ and $N$, we empirically select different combinations of $(M, N)$ pairs.

Radioprophet : we explore three off-the-shelf machine learning algorithms with the PerUserDynamic model ( $\alpha=1000$ and $\beta=1)$ : Naïve Bayes, Classification Tree, and Ensemble Bagging. Their performance and accuracy are summarized in Table 4 .

We now discuss the results in Table 5. "Fast dormancy 1s" is an aggressive approach incurring unacceptable signaling overhead. "Fast dormancy $3 \mathrm{~s}$ " reduces $\Delta(S)$ with less energy saving as expected. For both approaches, their $\Delta(E) /(1+\Delta(S))$ values

\footnotetext{
${ }^{2}$ We configured short screen timeout for the 20 phones so screen-off is good approximation for screen-idle.

${ }^{3}$ The performance numbers in Table 4 correspond to the execution time of the scripts written in Matlab on desktop. Our real implementation on the S3 smartphone uses $\mathrm{C}++$ so it is much more efficient $(\$ 5.6)$.
} 
Table 5. Comparison of optimization approaches. For RP, we use the PerUserDynamic model $(\alpha=1000, \beta=1)$ with setting $S_{5}$ in Table 3 RadioJockey is only applicable to screen-off traffic.

\begin{tabular}{|c|c|c|c|c|}
\hline Name & Description \& Configuration & $\Delta(E)$ & $\Delta(S)$ & $\frac{\Delta(E)}{(1+\Delta(S))}$ \\
\hline \hline Basic Fast dormancy 1s & Invoke fast dormancy after 1s idle time & $62.7 \%$ & $214.9 \%$ & 0.20 \\
\hline Basic Fast dormancy 3s & Invoke fast dormancy after 3s idle time & $40.9 \%$ & $95.8 \%$ & 0.21 \\
\hline \hline $\begin{array}{c}\text { RadioJockey } \\
\text { Assuming 100\% accuracy }\end{array}$ & $\begin{array}{c}\text { RadioJockey applied to } \\
\text { only screen-off traffic }\end{array}$ & $30.1 \%$ & $51.7 \%$ & $\begin{array}{c}0.20 \\
\text { (screen-off) }\end{array}$ \\
\hline $\begin{array}{c}\text { RadioJockey } \\
\text { Assuming 90\% accuracy }\end{array}$ & $\begin{array}{c}\text { RadioJockey applied to } \\
\text { only screen-off traffic }\end{array}$ & $27.2 \%$ & $52.0 \%$ & $\begin{array}{c}0.18 \\
\text { (screen-off) }\end{array}$ \\
\hline $\begin{array}{c}\text { MakeIdle } \\
\text { M:1000, N:100 }\end{array}$ & $\begin{array}{c}\text { MakeIdle: based on previous M packets, } \\
\text { predict next N packets }\end{array}$ & $64.9 \%$ & $305.2 \%$ & 0.16 \\
\hline $\begin{array}{c}\text { MakeIdle } \\
\text { M:10, N:10 }\end{array}$ & $\begin{array}{c}\text { MakeIdle: based on previous M packets, } \\
\text { predict next N packets }\end{array}$ & $44.9 \%$ & $195.2 \%$ & 0.15 \\
\hline \hline RP: Naïve Bayes & $\begin{array}{c}\text { Naïve Bayes classification with } \text { mvmn: } \\
\text { multivariate multinomial distribution }\end{array}$ & $53.0 \%$ & $107.9 \%$ & 0.25 \\
\hline RP: Classification Tree & Binary decision tree for classification & $59.1 \%$ & $91.0 \%$ & 0.31 \\
\hline RP: Ensemble Bagging & $\begin{array}{c}\text { Method: Bag; type: classification } \\
\text { weak leaner: decision tree; \# of trees: } 20\end{array}$ & $59.3 \%$ & $90.2 \%$ & 0.31 \\
\hline RP: Optimal & Predict all IBTs correctly & $59.8 \%$ & $85.4 \%$ & 0.32 \\
\hline
\end{tabular}

(the average radio energy saving per unit of signaling load) are low due to a lack of adaptation to dynamic traffic patterns.

For RadioJockey, by assuming the prediction accuracy for each background app to be $90 \%$, it saves $27.2 \%$ of radio energy with $52 \%$ of signaling load, which can be slightly improved when the accuracy increases to $100 \%$. The overall saving is lower than that of RP because RadioJockey does not handle foreground traffic usually triggered by user interaction ( 6 . For MakeIdle, we use two representative $(M, N)$ settings. In both cases, the incurred signaling load is prohibitive, since MakeIdle does not consider the very important signaling load metric in its optimization framework.

For RP, in the optimal case assuming $100 \%$ prediction accuracy, it saves $59.8 \%$ of radio energy with $85.4 \%$ of signaling load incurred. The signaling load is not zero, because for IBTs smaller than $T_{\text {tail }}$ but larger than SBT, even if the prediction is correct, invoking fast dormancy would still incur an extra state promotion. This is inherent for any fast dormancy based optimization technique. Among the three machine learning algorithms, Ensemble Bagging achieves the best results, likely due to its usage of multiple submodels to avoid overfitting. However, as shown in Table 4 its runtime overhead is very high. The Classification Tree approach achieves similar optimization results with much lower runtime overhead. The $\Delta(E) /(1+\Delta(S))$ metric indicates that RP outperforms other approaches in balancing $\Delta(E)$ and $\Delta(S)$.

\subsection{Running Overhead on Real Phone}

We implement the Radioprophet system on Android as discussed in $\$ 5.1$, in order to demonstrate its practicality on today's smartphones. We breakdown its runtime 
overhead into three components: (1) traffic monitoring and feature extraction, (2) model training and prediction, and (3) fast dormancy invocation. We found invoking fast dormancy incurs negligible overhead. We therefore focus on (1) and (2) below.

Traffic Monitoring and Feature Selection. Unlike RadioJockey requiring system call instrumentation, RP only needs to monitor packet traces, which is also needed by RadioJockey. On the S3 smartphone, our traffic monitor incurs no more than $1 \%$ of CPU overhead for parsing packet headers and generating burst features, although the overhead is much lower when the throughput is low (e.g., less than $200 \mathrm{kbps}$ ). The additional power to run the data collector is less than $17 \mathrm{~mW}$ most of the time. In contrast, the LTE radio power is at least $1000 \mathrm{~mW}$ [8].

Model Training and Prediction: Our implementation on S3 uses the Classification Tree model that balances between accuracy and performance (Table 4). We measure the average model training time to be $200 \mathrm{~ms}$ and the average prediction time to be $0.1 \mathrm{~ms}$. Its incurred power overhead is always negligible (less than $10 \mathrm{~mW}$ ).

\section{Related Work and Concluding Remarks}

We compare RP with three representative adaptive resource deallocation proposals.

TOP [10] leverages fast dormancy to eliminate the tail. It assumes each individual application can predict an imminent long IBT with reasonable accuracy, and fast dormancy is only invoked when the aggregate prediction across all concurrent apps is long enough. TOP provides the prediction framework, but it does not solve the challenging prediction problem itself, which is the key focus of RP.

MakeIdle [7] uses packet timing to calculate the optimal idle time before invoking fast dormancy, in order to maximize the radio energy saving. However, MakeIdle considers minimizing radio energy as the only objective, leading to unacceptably high signaling overhead shown in Table 5 It leaves the job of reducing the signaling load to another algorithm called MakeActive [7] that changes the traffic pattern by shifting packets. MakeActive does not work with foreground traffic that is usually delaysensitive, and even for background traffic, there is no guarantee that it does not affect user experience. In contrast, RP does not rely on changing traffic patterns and it works with both foreground and background traffic. It can in fact coexist with traffic shaping based optimization techniques such as MakeActive and TailEnder [5].

RadioJockey [4] uses program execution traces to predict the end of communication spurts and invoke fast dormancy when necessary. It however has several limitations. (1) It needs heavy instrumentation i.e., requiring complete system call traces in addition to packet traces, while RP only examines packet header information. (2) RadioJockey only works for background app without user interaction, since "predicting EOS events for foreground applications turns out to be challenging since user interactions can trigger network communications at any point in time" [4]. (3) RadioJockey treats different apps separately and does not predict start-of-session, hence when concurrent apps exist, the prediction accuracy would be affected. In contrast, RP introduces a general, lightweight, and effective framework that naturally optimizes concurrent traffic from both foreground and background apps. RP achieves even better optimization results for all traffic than RadioJockey does for only background traffic (Table 5). 
To conclude, we propose a novel, practical, and effective system called RadioProphet that intelligently predicts long idle period using off-the-shelf machine learning algorithms, and deallocate resources based on IBT prediction for cellular networks. Using 7-month data collected from 20 real users, we show that RP outperforms existing proposals in balancing the key tradeoff between resource saving and signaling load. We present the first implementation of adaptive resource deallocation using fast dormancy, demonstrating the feasibility of $\mathbf{R P}$ on real smartphones. We believe $\mathbf{R P}$ is an important step towards application-aware energy and resource optimization in wireless networks.

Acknowledgements. This research was supported in part by the National Science Foundation under grants CNS-1039657, CNS-1059372 and CNS-0964545.

\section{References}

1. UE "Fast Dormancy" behavior. 3GPP discussion and decision notes R2-075251 (2007)

2. Configuration of fast dormancy in release 8. 3GPP discussion notes RP-090960 (2009)

3. System Impact of Poor Proprietary Fast Dormancy. 3GPP discussion and decision notes RP090941 (2009)

4. Athivarapu, P., Bhagwan, R., Guha, S., Navda, V., Ramjee, R., Arora, D., Padmanabhan, V., Varghese, G.: RadioJockey: Mining Program Execution to Optimize Cellular Radio Usage. In: MobiCom (2012)

5. Balasubramanian, N., Balasubramanian, A., Venkataramani, A.: Energy Consumption in Mobile Phones: A Measurement Study and Implications for Network Applications. In: IMC (2009)

6. Breiman, L.: Bagging Predictor. Machine Learning 24(2) (1996)

7. Deng, S., Balakrishnan, H.: Traffic-Aware Techniques to Reduce 3G/LTE Wireless Energy Consumption. In: CoNEXT (2012)

8. Huang, J., Qian, F., Gerber, A., Mao, Z.M., Sen, S., Spatscheck, O.: A Close Examination of Performance and Power Characteristics of 4G LTE Networks. In: MobiSys (2012)

9. Huang, J., Qian, F., Mao, Z.M., Sen, S., Spatscheck, O.: Screen-Off Traffic Characterization and Optimization in 3G/4G Networks. In: Proc. ACM SIGCOMM IMC (2012)

10. Qian, F., Wang, Z., Gerber, A., Mao, Z.M., Sen, S., Spatscheck, O.: TOP: Tail Optimization Protocol for Cellular Radio Resource Allocation. In: Proc. ICNP (2010)

11. Qian, F., Wang, Z., Gerber, A., Mao, Z.M., Sen, S., Spatscheck, O.: Profiling Resource Usage for Mobile Applications: a Cross-layer Approach. In: MobiSys (2011) 